\title{
Variação do Módulo de Elasticidade de um solo colapsível de Petrolina-PE com e sem inundação
}

\author{
Variation of the modulus of elasticity of a \\ collapsible soil of Petrolina-PE with and \\ without flood
}

Jesce John da Silva Borges ${ }^{1}$, Marta Lúcia Rolim de Almendra Freitas ${ }^{2}$, Silvio Romero de Melo Ferreira ${ }^{1}$

\footnotetext{
${ }^{1}$ Universidade Federal de Pernambuco - UFPE, CEP 50740-530, Recife, PE e-mail: jescejohn@hotmail.com, sr.mf@hotmail.com

${ }^{2}$ Universidade Católica de Pernambuco - UNICAP, CEP 50050-900, Recife, PE e-mail: marta.rolim@uol.com.br
}

\begin{abstract}
RESUMO
Solos colapsíveis são solos não saturados que experimentam um rearranjo radical de partículas e grande redução de volume quando inundados, com ou sem aplicação carga adicional. Esses solos apresentam sérios problemas para a Engenharia Geotécnica quando há mudança da umidade, causando deformações adicionais aos solos. A análise do seu comportamento passa a ser uma etapa essencial para o sucesso do projeto de engenharia. Ensaios edométricos simples e duplos, com sucção controlada e a utilização do Expansocolapsômetro têm sido bastante utilizadas para analisar a variação de volume dos solos colapsíveis devido à mudança de umidade, pois permitem avaliar o comportamento dos solos colapsíveis de modo que simulem as condições a que o solo será submetido. $O$ artigo tem por objetivo avaliar o módulo de elasticidade em campo de um solo colapsível de um conjunto Residencial em Petrolina-PE, com e sem a inundação prévia. Foi utilizado em campo o Light Weight Deflectometer - LWD que se trata de um equipamento moderno que aplicação carga dinâmica em uma placa ao solo e permite medir as deflexões e quantifica o módulo de elasticidade. A caracterização física do solo foi realizada em laboratório. O resultado apresentou um solo preponderantemente arenoso, com um módulo de elasticidade que varia de 46,3 a 79,2 $\mathrm{MPa}$ e a inundação causa uma redução de $88 \%$. Além disso, verificou-se que o aumento da umidade associada à aplicação da carga dinâmica causa uma redução de sua rigidez, ocorrendo uma diminuição do módulo de elasticidade e, consequentemente, um aumento da deflexão. A partir dos resultados do LWD, estabeleceu-se um critério de identificação de solo colapsível através do coeficiente $\mathrm{K}_{\mathrm{LWD}}$, atestando que o solo de Petrolina apresenta suscetibilidade ao colapso: alto para $\mathrm{K}_{\mathrm{LWD}}>4,2$, baixo para $\mathrm{K}_{\mathrm{LWD}}<2,8$ e médio para $\mathrm{K}_{\mathrm{LWD}}$ entre 2,8 e 4,2.
\end{abstract}

Palavras-chave: Solo Colapsível, Solo Não Saturado, Módulo de Elasticidade, Light Weight Deflectometer.

\section{ABSTRACT}

Collapsible soils are unsaturated soils that undergo radical rearrangement of particles and great reduction of volume when wetting, with or without additional load application. These soils present serious problems for Geotechnical Engineering when there is a change of water content, causing additional deformations to the soils. The analysis of their behavior becomes an essential step for the success of an engineering project. Single and double edometric tests with controlled suction and the use of the Expanso-colapsometer were widely used to analyze the volume variation of the collapsible soils due to the change of water content, since they allow to evaluate the behavior of the collapsible soils in a way that simulate the conditions to which the soil will be submitted. The objective of this paper is to evaluate the elastic modulus in field of a collapsible soil of a residential complex in Petrolina-PE, with and without previous wetting. It was used in the field the Light Weight Deflectometer - LWD that is modern equipment that application load dynamic in a plate to the ground and allows measuring the deflections and quantifies the modulus of elasticity. The physical characterization of the soil was performed in the laboratory. The result showed a predominantly sandy soil with a modulus of elasticity ranging from 46.3 to $79.2 \mathrm{MPa}$ and the wetting causes a reduction of $88 \%$. In 
addition, it was found that the increase of moisture associated with the application of the dynamic load causes a reduction of its stiffness, occurring a decrease of the modulus of elasticity and, consequently, an increase of the deflection. From the results of the LWD, a criterion of identification of collapsible soil was established through the $\mathrm{K}_{\mathrm{LWD}}$ coefficient, attesting that Petrolina's soil presents the following susceptibility to collapse: high for $\mathrm{K}_{\mathrm{LWD}}>4.2$, low for $\mathrm{K}_{\mathrm{LWD}}<2.8$ and medium For $\mathrm{K}_{\mathrm{LWD}}$ between 2.8 and 4.2.

Keywords: Collapsible Soil, Unsaturated Soil, Elasticity Modulus, Light Weight Deflectometer.

\section{INTRODUÇÃO}

Uma definição para solos colapsíveis se deu em 1976, na convenção anual da American Society of Civil Engineers (ASCE), na Filadélfia, estabelecendo que o solo colapsível é definido como "o solo não saturado que experimenta um rearranjo radical de partículas e grande redução de volume quando inundado, com ou sem carga adicional" [1].

Os solos colapsíveis podem ser considerados como: "Solos Verdadeiramente Colapsíveis" quando eles colapsam sobre peso próprio das terras quando inundado e "Solos Condicionados ao Colapso", quando eles são capazes de suportar certo nível de tensão após saturação [2].

Solos colapsíveis são encontrados em muitas partes do mundo, Estados Unidos da América, Brasil, Argentina, Uruguai, Espanha, Austrália, Kuwait, Egito, Irã, África do Sul e China, particularmente em regiões áridas, semi-áridas e tropicais [3, 4]. A maioria dos solos colapsíveis encontrados são formados de depósitos eólicos e aluvionares $[5,6,7,8]$. Solos residuais que sofrer um processo pedogênico o que resulta em um solo estruturalmente instável e com macroporos [1,9].

No Brasil, ocorrem em diversos Estados: Amazonas, Piauí, Ceará, Pernambuco, Bahia, Minas Gerais, Tocantins, São Paulo, Paraná, Santa Catarina e Rio Grande do Sul e no Distrito Federal. Solos colapsíveis já foram encontrados em Petrolina-PE em vários locais associados a obras de infraestruturas e conjuntos habitacionais: $[10,11,12,13,14,15]$. Cerca de $50 \%$ dos solos superficiais do município apresentam suscetibilidade alta ou média de ocorrência desses solos [13]. Vários danos (rachaduras e trincas) já foram causados nessas edificações com prejuízo socioeconômico.

A identificação de solos colapsíveis significa uma etapa essencial no sucesso de projetos de engenharia, pois falhas nessa etapa podem levar à ruptura de obras ou à necessidade de recuperação. Existem métodos tradicionais de identificação de solos colapsíveis. Ferreira [5] classificou os métodos em indiretos e diretos. Os métodos indiretos utilizam índices físicos, gramulometria, cartografias geotécnicas (orientativos) para a identificação e os diretos avaliam os colapsos através dos ensaios edométricos convencionais e com sucção controlada, Expansocolapsômetro e em placa. No presente artigo foi utilizado um equipamento moderno que vem ganhando espaço no cenário do desenvolvimento técnico da engenharia, o Light Weight Deflectometer LWD. Refere-se a um ensaio dinâmico de carregamento com placa, em que a força de impacto é gerada pela queda de um peso sobre um sistema de amortecedores, transmitindo impulso ao solo através de uma placa de carga.

A medição da força no LWD é feita através de uma célula de carga instalada junto da placa e a medição das deflexões em vários pontos da superfície é realizada por um sistema de três geofones que medem as velocidades que, por integração no tempo, fornecem as deflexões ocorridas na superfície ensaiada. As informações dos registros da força aplicada e a deflexão dos pontos são transmitem a um computador portátil, [16,17]. Os dados são interpretados utilizando recurso da equação da deflexão num meio espaço sujeito a uma carga, derivada da teoria de Boussinesq. A deflexão no centro da placa de carga é utilizada para calcular o módulo de elasticidade ou de resiliência da camada de estudo, [18]. O LWD tem suas maiores aplicações no controle de compactação em obras rodoviárias, $[17,18]$. Buscou-se no presente artigo utilizar o equipamento para avaliar as deflexões que ocorrem em um solo colapsível na umidade natural e previamente inundado.

O resultado dos ensaios edométricos duplos obtido para avaliar a colapsibilidade do solo é de natureza diferente do resultado obtido dos ensaios com o LWD. Um é estático, o outro é dinâmico; um mede deformação e potencial de colapso, o outro deflexão; um mobiliza a espessura do corpo de 20 a $30 \mathrm{~mm}$, o outro mobiliza uma espessura de $400 \mathrm{~mm}$, um é método direto de investigação, o outro é indireto; um requer coleta de amostra, o outro é realizado em campo, um requer um tempo de ensaio bem mais longo, o outro alguns minutos (cerca de 60 mimutos para realizar 8 determinações); um é um ensaio quantitativo, o outro é indicativo. É importante destacar que a inundação prévia em ambos ensaios antes do carregamento (estático ou dinâmico) provoca mudança no estado tensional, modificando a sucção e rigidez, [19,20].

Os danos nas edificações podem ser evitados se na fase do projeto os solos colapsíveis forem identificados de 
forma adequada, avaliando-se as deformações adicionais e a redução de capacidade de suporte do solo devido à variação de umidade. Este artigo avaliou o módulo de elasticidade devido à mudança de umidade em um solo colapsível, aluvionar, do nordeste do Brasil, no Semi-árido de Pernambuco, na cidade de Petrolina. Além disso, estabelece um critério de suscetibilidade ao colaspo através do ensaio com o LWD.

\section{MATERIAIS E MÉTODOS}

O programa de investigação geotécnica ocorreu em um local próximo a um conjunto habitacional do Programa do Governo Federal Minha Casa Minha Vida no município de Petrolina-PE. O solo encontra-se em uma área suscetibilidade alta de ocorrência de solo colapsível [13], é essencialmente quartzoso com textura arenosa, não líquido e não plástico, se enquadragrupo SM, areia siltosa, na classificação do SUCS, [15]. Tem permeabilidade da ordem de $10^{-6} \mathrm{~m} / \mathrm{s}$ É verdadeiramente colapsível $[12,14]$ e apresenta comportamento de pico na relação entre o potencial de colapso e a tensão aplicada, com valor máximo de 3,57\% na tensão de $160 \mathrm{kPa}$, [15]. Em campo foi utilizado o Light Weight Deflectometer - LWD para avaliar o módulo dinâmico de elasticidade em solo natural e inundado. Já em laboratório, foi realizada a caracterização física do solo (umidade, peso específico natural e granulometria).

$\mathrm{Na}$ caracterização física do solo a preparação das amostras seguiu as recomendações da ABNT [21] e foram realizados os seguintes ensaios: Análise granulométrica, ABNT [22] e Massa específica dos grãos do solo, ABNT [23].

\subsection{Determinação do módulo dinâmico de elasticidade avaliado pelo Light Weight Deflectometer - LWD}

O módulo dinâmico de elasticidade foi obtido por meio de ensaio dinâmico de carregamento com placa através do equipamento Light Weight Deflectometer - LWD, Figura 1a. Os ensaios foram realizados em duas áreas com dimensões de 2,0 m x 2,0 m, conforme apresentada na Figura 1b. Na Área A foi realizado no solo na umidade natural a $0,30 \mathrm{~m}$ de profundidade e na Área B a 0,05 m de profundidade, sendo o solo inundado previamente de forma lenta e progressiva de modo a se obter uma umidade uniforme próxima à umidade de saturação, em uma espessura de $0,40 \mathrm{~m}$ (profundidade de influência do carregamento do impacto) [24], avaliada antes da realização do ensaio (Figura 2). Nas Áreas A e B foram realizados 32 ensaios no total, sendo que para cada área, 8 (oito) ensaios foram realizados com carga de impacto de $10 \mathrm{kgf}$ e, em seguida, mais 8 (oito) ensaios foram realizados com carga de impacto de $15 \mathrm{kgf}$.

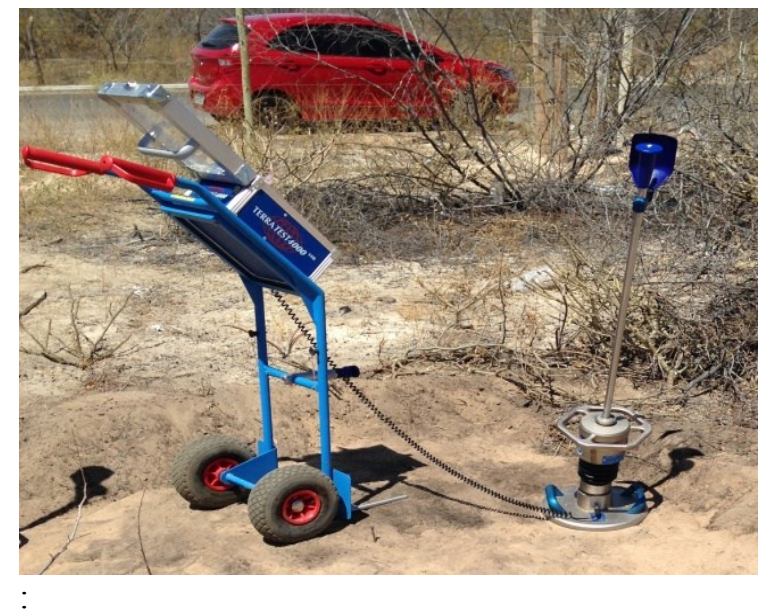

(a)

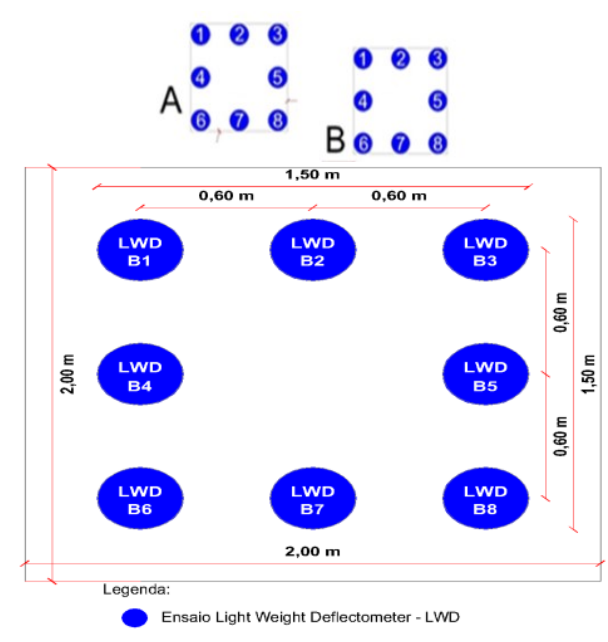

(b)

Figura 1: Ensaio com o LWD: (a) TERRATEST 4000 USB e (b) representação esquemática dos ensaios de campo nas áreas $\mathrm{A}$ e $\mathrm{B}$, foram numerados de 1 a 8 os ensaios com o LWD para cada área.

A carga de impacto é causada pela queda de um peso sobre uma placa com diâmetro de 0,30 m, o que gera uma força máxima $\left(\mathrm{F}_{\text {máx }}\right)$ de $7,070 \mathrm{kN}$ e $10,6 \mathrm{kN}$ para os pesos de $10 \mathrm{kgf}$ e de $15 \mathrm{kgf}$, respectivamente. A altura de queda do peso é de $0,715 \mathrm{~m}$. Durante a calibração do dispositivo, esta força é ajustada de modo que a tensão normal $\left(\sigma_{\text {máx }}\right)$ sob a placa seja de $0,1 \mathrm{MN} / \mathrm{m}^{2}$ para o peso de $10 \mathrm{kgf}$ e $0,15 \mathrm{MN} / \mathrm{m}^{2}$ para o peso de 15 kgf. Segundo a TP BF-StB Part B 8.3 [24], o módulo de elasticidade $\mathrm{E}_{\mathrm{LwD}}$ é um parâmetro de 
deformabilidade do solo sob uma carga de impacto vertical e seu valor é calculado em função da amplitude dos deslocamentos medidos de acordo com a Equação 1.

$$
\mathrm{E}_{\mathrm{LWD}}=1,5 \mathrm{r} \frac{\sigma_{\text {máx }}}{\mathrm{s}_{\text {máx }}}
$$

Onde: $\mathrm{s}_{\text {máx }}$ significa o valor médio das três últimas deflexões máximas obtidas após os três testes de précarregamento, r é o raio da placa de carga $(0,15 \mathrm{~m})$ e $\sigma_{\operatorname{máx}}$ é a tensão normal máxima sob a placa de carga $(0,1$ $\mathrm{MN} / \mathrm{m}^{2}$ ou $0,15 \mathrm{MN} / \mathrm{m}^{2}$ ) em contato com o solo.

O equipamento LWD é muito utilizado para medir as deflexões nas camadas compactas das bases dos pavimentos. Além do módulo de elasticidade, informa a deflexão média (s), a velocidade média referente aos três últimos impactos (v) e o grau de compactabilidade (s/v). Se o valor de $\mathrm{s} / \mathrm{v}$ for $>3,5$ o solo compactado necessita de compactação adicional ou ser substituído por outro [24].

O ensaio foi realizado com o seguinte procedimento: a placa de carga é colocada ao solo de modo a garantir o pleno contato; o dispositivo de carregamento é colocado no centro da placa e acoplado ao computador de teste; verifica-se a verticalidade e o ensaio pode ser inicializado. A carga de impacto é solta em queda livre de uma altura de $0,715 \mathrm{~m}$ por 03 (três) vezes referentes ao pré-carregamento e 03 (três) quedas referentes ao ensaio propriamente dito, medindo-se as deflexões.

O TERRATEST USB 4000 apresenta um programa que permite realizar tanto uma análise estatística dos resultados, como também emitir um relatório em pdf. A determinação do módulo de elasticidade é feita conforme a norma alemã [24]. O programa também gera relatórios individuais de cada ensaio mostrando detalhes das curvas de deflexão, permite incluir uma foto do ensaio e ainda apresentar a localização do ensaio no mapa através de Google Maps.

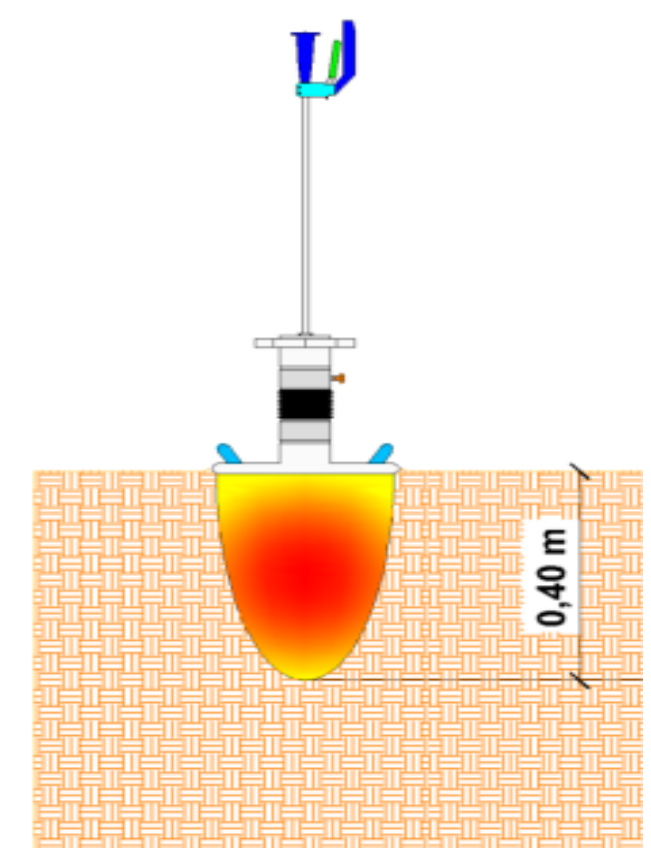

Figura 2: Representação esquemática da faixa de profundidade alcançada pelo ensaio com o LWD.

O perfil do solo de Petrolina até 9 metros de profundidade é constituído de duas camadas, uma areia fina siltosa de pouco a medianamente compacta seguida de uma areia fina e média de medianamente compacta a compacta, Figura 3a. Considerando os valores médios o índice de resistência à penetração (golpes $/ 0,3 \mathrm{~m}$ ) cresce com a profundidade $\left(\mathrm{N}_{\mathrm{SPT}}=1,5 \mathrm{Z}+3,6 \mathrm{com} \mathrm{R} \mathrm{R}^{2}=0,91 \mathrm{e} \mathrm{Z} \mathrm{em} \mathrm{m}\right.$ ), Figura 3b. A 9,5 $\mathrm{m}$ o impenetrável a percussão é atingido e o solo é um pedregulho com argila siltosa, [15]. 


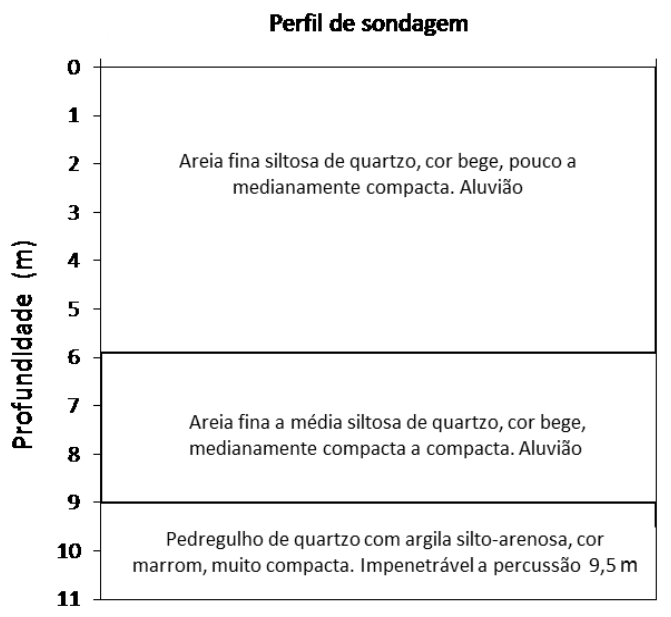

(a)

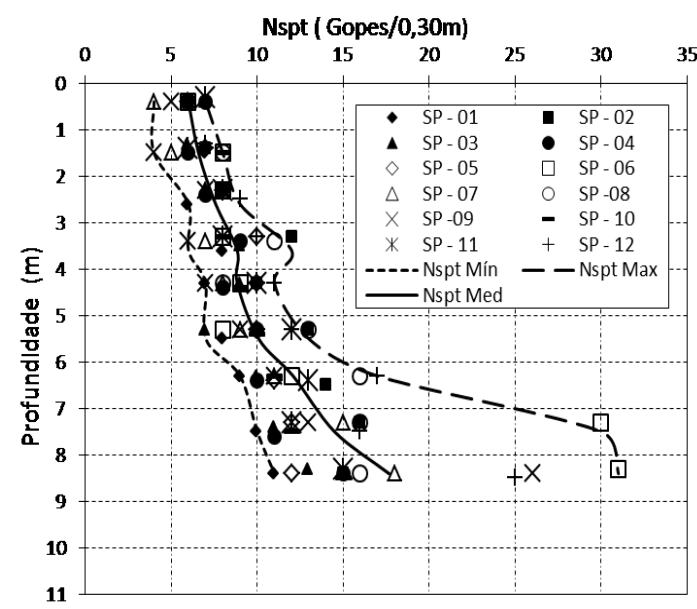

(b)

Figura 3: Perfil do solo, [15].

\section{RESULTADOS E DISCUSSÃO}

\subsection{Caracterização Física}

O solo é essencialmente arenoso com mais de $90 \%$ de areia. A curva granulométrica obtida do solo com e sem defloculante é apresentada na Figura 4. Praticamente não apresenta silte (1\%) e possui fração argilosa igual a 5\%. Percebe-se que, no ensaio sem defloculante, a porcentagem de areia fina cresce enquanto a de argila decresce, Tabela 1 . O peso específico natural do solo varia de 15,85 a $17,48 \mathrm{kN} / \mathrm{m}^{3}$, a umidade natural é em torno de $0,80 \%$ e grau de saturação de $2,60 \%$.

Tabela 1: Resultado do ensaio com e sem defloculante.

\begin{tabular}{ccccccc}
\hline Ensaio & Pedregulho & Areia grossa & Areia média & Areia fina & Silte & Argila \\
\hline Com defloculante & 0 & 0 & 06 & 88 & 01 & 05 \\
\hline Sem defloculante & 0 & 0 & 06 & 92 & 0 & 02 \\
\hline
\end{tabular}

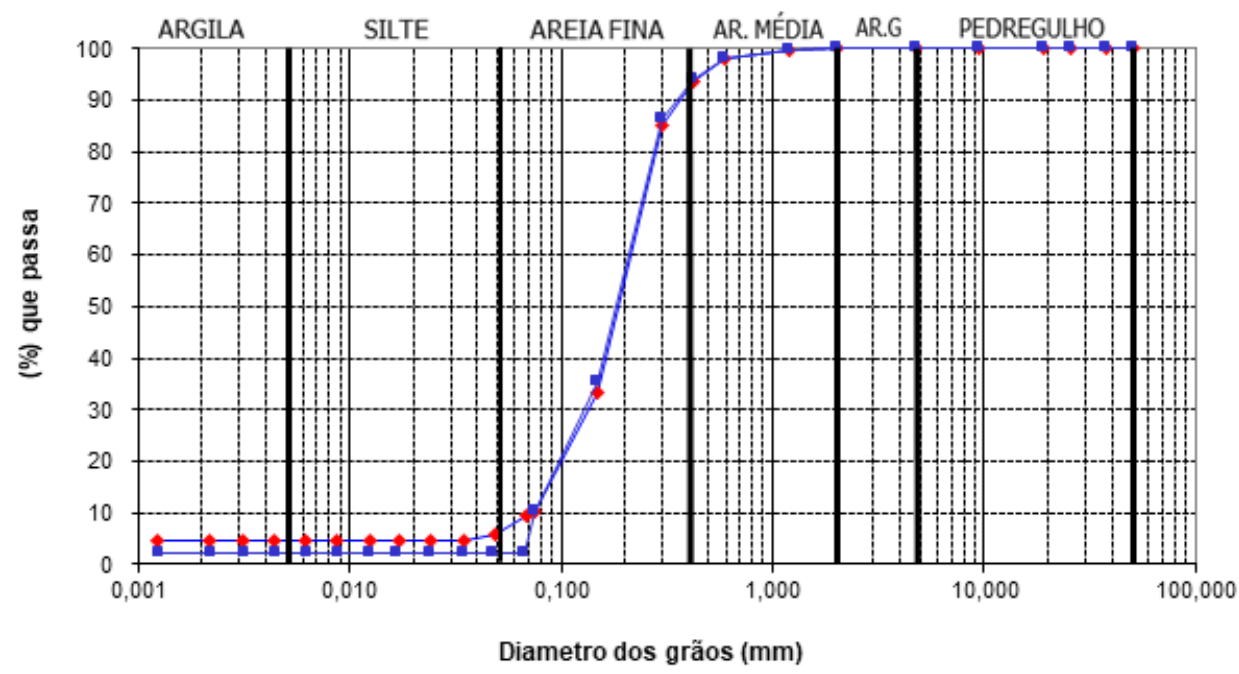

$\multimap-$ Com defloculante $\quad-$ Sem defloculante

Figura 4: Curva granulométrica com e sem defloculante. 


\subsection{Determinação do Módulo de Elasticidade}

A Figura 5 mostra as deflexões após impacto do peso sobre a placa no solo na umidade natural e inundado previamente. No solo natural, as curvas individuais são sobrepostas (Figura 5a) enquanto que para o solo previamente inundado há distanciamento das 3 curvas individuais; assim como constata-se os maiores valores das deflexões (Figura 5b). As deflexões no solo inundado previamente são 7,7 vezes maiores que as deflexões no solo natural. O grau de compactabilidade $(\mathrm{s} / \mathrm{v})$ no o solo natural é superior a 3 vezes a do solo inundado. Solos fofos ou poucos resistentes apresentam baixos valores de módulo de elasticidade e as três curvas individuais de deflexão apresentam afastamentos entre si [25]. O solo com umidade natural muito baixa $(0,72 \%)$, associado a um módulo de elasticidade maior indica uma maior rigidez e consequentemente menores deflexões, a inundação eleva a umidade, reduz a sucção, reduz o módulo de elasticidade e eleva as deflexões que crescem a medida que as cargas por impactos são aplicadas. Portanto, através da análise das curvas de deflexões, é possível perceber o comportamento do solo quanto à perda da resistência quando submetido à inundação e aplicação de carga.

A Tabela 2 apresenta os valores dos módulos de elasticidade (ELWD) obtidos com o LWD. O solo previamente inundado apresenta valores bem menores que o solo na umidade natural, enquanto que as deflexões (s médio) apresentaram valores superiores. O módulo de elasticidade no solo natural varia de 46,3 a 79,2 MPa, enquanto no solo inundado, varia de 9,3 a 22,5 MPa. A inundação causa uma redução de $88 \%$ do nos valores dos módulos de elasticidade.

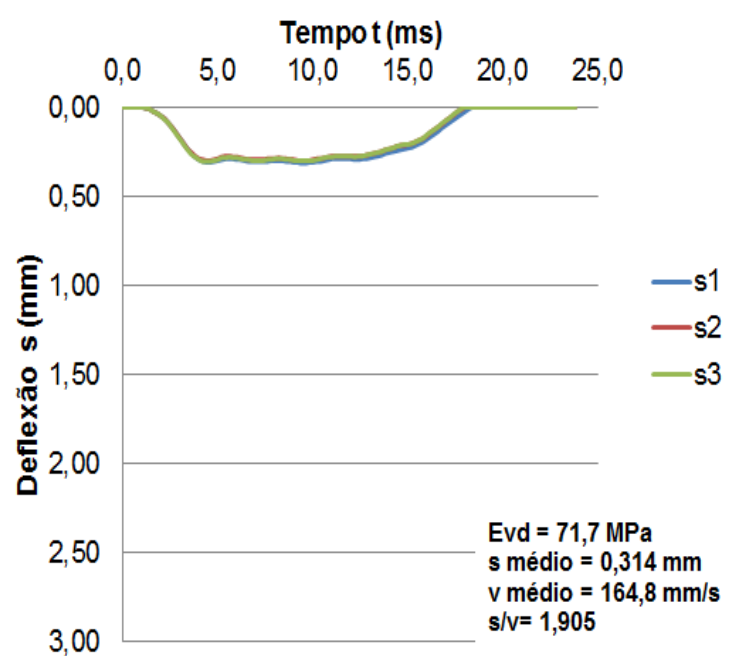

(a) Solo na umidade natural

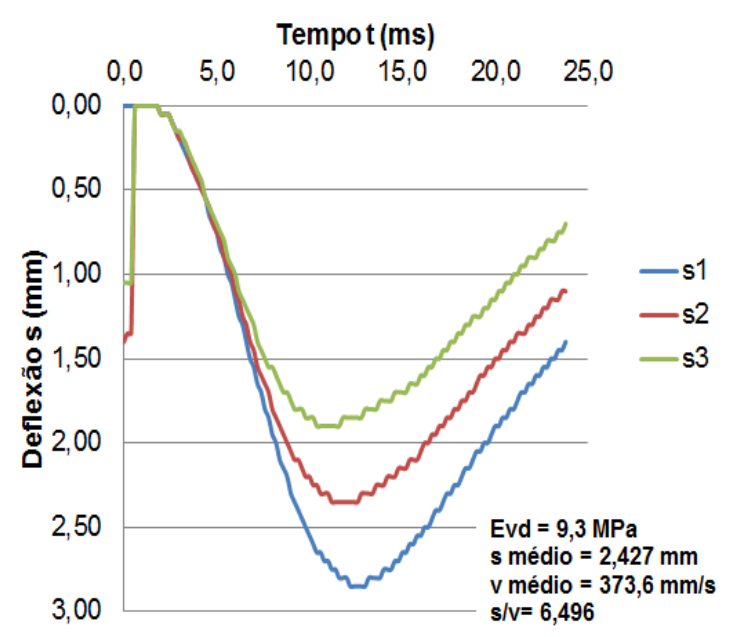

(b) Solo inundado previamente

Figura 5: Deflexões individuais para o solo na umidade natural e previamente inundado, ensaio realizado com peso de 10 kgf: (a) Ponto 6 da Área A e (b) Ponto 5 da Área B.

Tabela 2: Módulos dinâmico de elasticidade $\left(\mathrm{E}_{\mathrm{LWD}}\right)$, deflexões (s) e grau de compactibilidade (s/v) obtidos com o LWD para a situação do solo na condição natural e inundado nas áreas A e B utilizando peso de $10 \mathrm{kgf}$ e $15 \mathrm{kfg}$

\begin{tabular}{|c|c|c|c|c|c|}
\hline Local & Peso & Valores & $\mathrm{E}_{\mathrm{LWD}}(\mathrm{MPa})$ & S médio $(\mathrm{mm})$ & $\mathrm{s} / \mathrm{v}(\mathrm{ms})$ \\
\hline \multirow{3}{*}{$\begin{array}{c}\text { Área A } \\
\text { (natural) }\end{array}$} & \multirow{3}{*}{$10 \mathrm{kgf}$} & Médio & 59,3 & 0,391 & 2,156 \\
\hline & & Mínimo & 46,3 & 0,284 & 1,872 \\
\hline & & Máximo & 79,2 & 0,486 & 2,349 \\
\hline \multirow{3}{*}{$\begin{array}{c}\text { Área B } \\
\text { (inundado) }\end{array}$} & \multirow{3}{*}{$10 \mathrm{kgf}$} & Médio & 16,1 & 1,509 & 5,425 \\
\hline & & Mínimo & 9,3 & 1,000 & 3,907 \\
\hline & & Máximo & 22,5 & 2,427 & 6,496 \\
\hline \multirow{3}{*}{$\begin{array}{c}\text { Área A } \\
\text { (natural) }\end{array}$} & \multirow{3}{*}{$15 \mathrm{kgf}$} & Médio & 55,8 & 0,616 & 2,256 \\
\hline & & Mínimo & 46,4 & 0,472 & 1,958 \\
\hline & & Máximo & 71,5 & 0,727 & 2,604 \\
\hline \multirow{3}{*}{$\begin{array}{c}\text { Área B } \\
\text { (inundado) }\end{array}$} & \multirow{3}{*}{$15 \mathrm{kgf}$} & Médio & 18,3 & 1,888 & 5,227 \\
\hline & & Mínimo & 14,7 & 1,480 & 4,281 \\
\hline & & Máximo & 22,8 & 2,297 & 5,786 \\
\hline
\end{tabular}

A Figura 6 mostra a variação dos valores das deflexões após impacto, utilizando o peso de 10,0 kgf e posteriormente o peso de 15,0 kgf na condição de umidade natural (Figura 6a) e no solo inundado 
previamente (Figura 6b). Observa-se que há um aumento das deflexões no solo com a umidade natural e consequentemente diminuição do módulo de elasticidade. Para um mesmo esforço (peso) a inundação causa um acréscimo das deflexões e para uma situação de umidade o acréscimo dos esforços causa maior deflexão com era de se esperar. No solo natural, o acréscimo nos valores das deflexões foi de $57,54 \%$, enquanto que no solo inundado previamente o acréscimo foi menor, $25,12 \%$. Isto pode ser explicado pelo fato de que solo inundado previamente sob o impacto da carga de $10 \mathrm{kgf}$ ter apresentado deflexões maiores (devido a carga e a inundação) do que no solo na umidade natural quando recebeu o impacto da carga de 15 kgf. No solo natural o grau de compactabilidade $(\mathrm{s} / \mathrm{v})$ apresenta um pequeno crescimento $(4,6 \%)$ e no solo inundado previamente apresenta uma pequena diminuição $(3,7 \%)$. O valor da relação $(\mathrm{s} / \mathrm{v})$ para as duas cargas de impacto na condição do solo previamente inundada foi $>3,5$ o que indica que o solo precisa ser mais compactado ou substituído por outro [25].

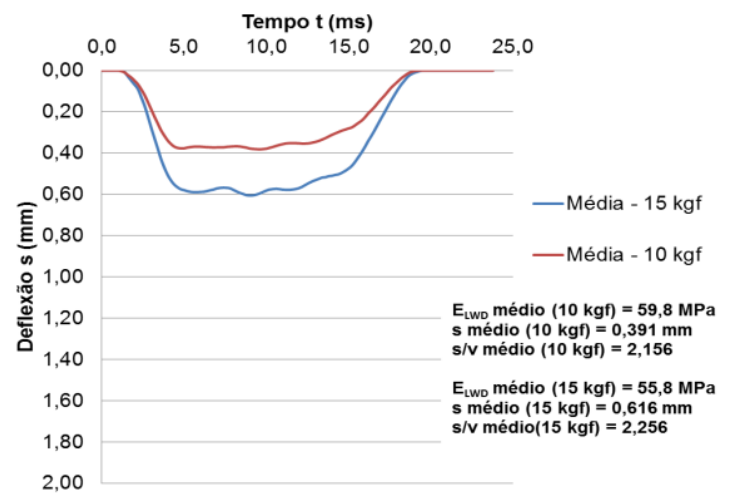

(a)

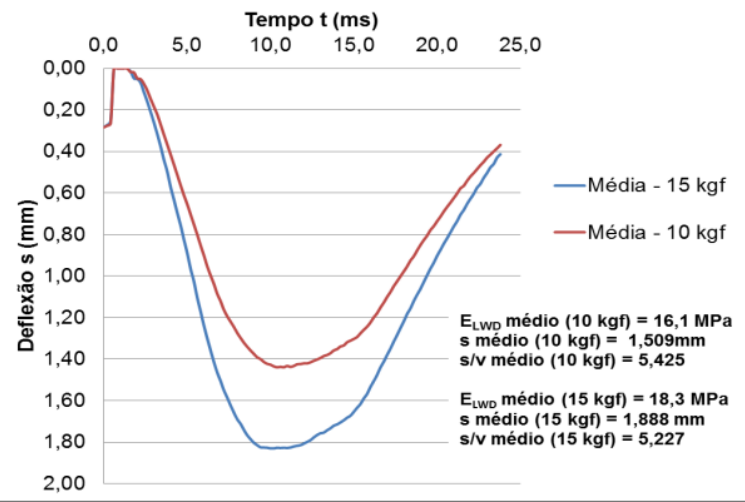

(b)

Figura 6: Deflexões devidas aos pesos de $10 \mathrm{kgfe} 15 \mathrm{kgf}$ (a) Área A, solo natural e (b) Área B, solo inundado.

Com o objetivo de analisar o comportamento do solo após a repetição dos ensaios no mesmo local, foram executados na Área A e na Área B quinze impactos com o peso de $15 \mathrm{kgf}$ e, em seguida, realizou-se um novo ensaio. Os resultados apontaram um aumento considerável nos módulos de elasticidade, sendo um aumento de 67,64 \% para a Área A (solo natural), (Figura 7a), e de 59,42\% para a Área B (solo inundado), (Figura 7b). Também se observou a sobreposição das curvas de deflexões individuais, passando a visualizarse uma única curva de deflexão. Este fato mostra que o solo necessita de compactações adicionais e que a aproximação das curvas de deflexão se deu devido à energia do ensaio que diminuiu a deformação após as sucessivas aplicações de tensão, ou seja, os quinze impactos resultaram na compactação do solo. Quanto ao grau de compactabilidade, os valores de s/v passaram a se aproximar do valor de 3,5. O comportamento é semelhante ao descrito por Fleming et. al [26], em que os ensaios repetidos resultam na compactação do solo com a energia do ensaio, diminuindo a deformação registrada ao longo das sucessivas aplicações de tensão.

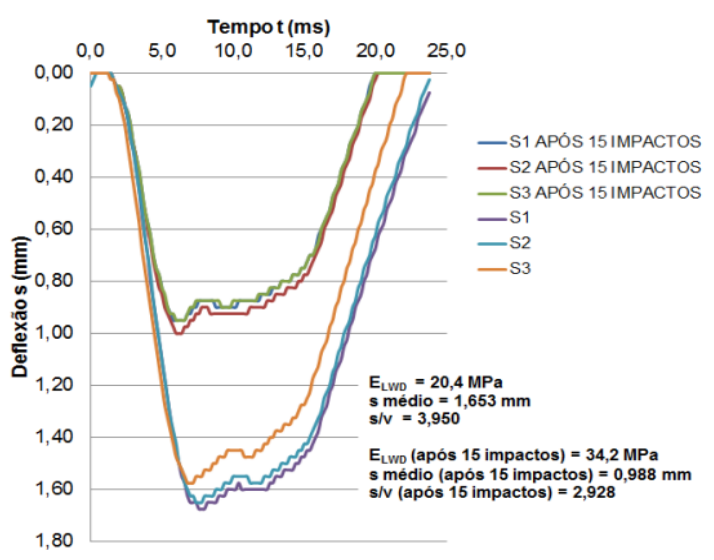

(a)

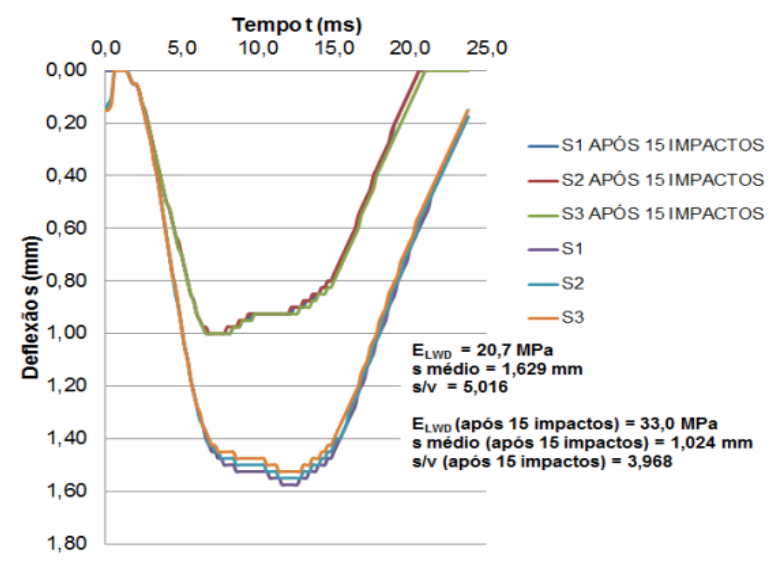

(b)

Figura 7: Análise dos resultados após 15 impactos com o peso de 15 kgf: (a) Área A, solo natural e (b) Área B, solo inundado. 
O coeficiente de colapsibilidade $\left(\mathrm{K}_{\mathrm{LWD}}\right)$ definido como a relação entre os módulos de elasticidade obtidos com o LWD, no solo natural e no solo previamente inundado, permite relacionar a instabilidade volumétrica. A Tabela 3 apresenta os resultados dos coeficientes $\mathrm{K}_{\mathrm{LWD}}$ obtidos pela Equação (2) a partir dos valores do módulo de elasticidade encontrados da Área A e na Área B, utilizando as cargas de impacto de 10 kgf e $15 \mathrm{kgf}$. Verificou-se maiores valores do $\mathrm{K}_{\mathrm{LWD}}$ para a carga de impacto de $10 \mathrm{kgf}$ e esse comportamento é devido ao fato que, antes do ensaio com a carga de $15 \mathrm{kgf}$, o solo já havia sido comprimido e colapsado sob a carga de10 kgf. Para estabelecer as faixas de valores representadas por cada nível de suscetibilidade, realizou-se a média dos 8 menores valores de $\mathrm{K}_{\mathrm{LWD}}$ e dos 8 maiores. Para este, foi atribuída a suscetibilidade alta para valores acima da respectiva média, enquanto aquele, foi atribuída a suscetibilidade baixa para valores abaixo da respectiva média e, por fím, foi atribuída a suscetibilidade média para aos valores intermediários. Dessa forma, a faixa de valores de $\mathrm{K}_{\mathrm{LWD}}$ e seus níveis de suscetibilidade estão apresentados na Tabela 4.

$$
\mathrm{K}_{\mathrm{LWD}}=\frac{\mathrm{Eq}}{\mathrm{EW}}
$$

onde:

$\mathrm{K}_{\mathrm{LWD}}=$ coeficiente de colapso obtido com LWD;

$\mathrm{Eq}=$ módulo de elasticidade do LWD no solo natural, em MPa;

$\mathrm{Ew}=$ módulo de elasticidade do LWD no solo inundado, em MPa.

Tabela 3: Valores de $\mathrm{K}_{\mathrm{LWD}}$.

\begin{tabular}{|c|c|c|}
\hline Ponto de determinação & Área A e B (10 kgf) $K_{L W D}$ & Área A e B (15 kgf) $K_{L W D}$ \\
\hline 1 & 3,10 & 2,76 \\
\hline 2 & 2,90 & 3,10 \\
\hline 3 & 4,48 & 3,35 \\
\hline 4 & 4,95 & 3,79 \\
\hline 5 & 4,99 & 3,22 \\
\hline 6 & 4,33 & 2,76 \\
\hline 7 & 4,13 & 3,64 \\
\hline 8 & 2,24 & 2,17 \\
\hline
\end{tabular}

Tabela 4: Identificação da colapsividade em solos do município de Petrolina-PE, através do ensaio LWD.

\begin{tabular}{c|c}
\hline Faixa de valores de $\mathrm{K}_{\mathrm{LWD}} *$ & Nível de Suscetibilidade ao colapso \\
\hline $\mathrm{K}_{\mathrm{LWD}}<2,8$ & Baixo \\
$2,8<\mathrm{K}_{\mathrm{LWD}}<4,2$ & Médio \\
$\mathrm{K}_{\mathrm{LWD}}>4,2$ & Alto \\
\hline
\end{tabular}

*Valores obtidos em região de alta suscetibilidade ao colapso com base na carta elaborada por Silva [13].

Os valores do $\mathrm{K}_{\mathrm{LWD}}$ são maiores para a carga de impacto de $10 \mathrm{kgf}$ do que para a carga de $15 \mathrm{kgf}$. Deve-se ao fato de que o solo já havia sofrido uma compressão e colapso sob a carga de10 kgf quando a carga de $15 \mathrm{kgf}$ foi aplicada.

\section{CONCLUSÕES}

O módulo de elasticidade varia de 46,3 a $79,2 \mathrm{MPa}$ e a inundação causa uma redução de $88 \%$ desse parâmetro. As deflexões no solo inundado são 7,7 vezes maiores que as deflexões no solo natural.

$\mathrm{O}$ coeficiente de colapsibilidade $\left(\mathrm{K}_{\mathrm{LWD}}\right)$ definido pela relação entre os módulos de elasticidade obtidos com o LWD, no solo natural e no solo previamente inundado é um indicador da suscetibilidade ao colapso. Indicando alta para $\mathrm{K}_{\mathrm{LWD}}>4,20$, média para $\mathrm{K}_{\mathrm{LWD}}$ entre 2,8 e 4,20 e baixa para $\mathrm{K}_{\mathrm{LWD}}<2,8$.

A aplicação do equipamento LWD permite identificar solos colapsíveis superficiais. Demonstrou ser uma técnica que avaliar a perda do módulo de elasticidade solo devido à inundação. Tem praticidade na 
BORGES, J.J.S.; FREITAS, M. L.R.A.; FERREIRA, S. R.M. revista Matéria, Suplemento, 2017.

execução e obtenção de resultados imediatos sem necessidade de cálculos posteriores.

Os resultados obtidos com o LWD mostram que o aumento da umidade associada à aplicação da carga dinâmica causa uma redução no módulo de elasticidade, aumento da deflexão e, consequentemente diminuição da rigidez.

\section{AGRADECIMENTO}

À empresa SEFE - Serviços Especiais de Fundações e Estruturas LTDA, representada por André Campelo de Melo e Henrique Soares de Azevedo de Melo, por ter cedido gentilmente o equipamento Light Weight Deflectometer - LWD, usado no estudo de caso deste trabalho.

\section{BIBLIOGRAFIA}

[1] CLEMENCE, S.P., FINBARR, A.O. "Design considerations for collapsible soils", Journal of the Geotechnical Engineering Division, v.7, n. GT3, pp. 305-317.1981.

[2] REGINATTO, A.R, FERRERO, J.C. "Colapse potencial of soils and soil-water chemistry", In: Proc. of the 8th I.C.S.M.F.E., Moscow, 22, pp.177-183, 1973.

[3] DUDLEY, J.H. "Review of collapsing soils", Journal of Geotechnical Engineering Division, v. 96, n. 5, pp. 925-947, 1970.

[4] AL-RAWAS, A.A. "State-of-the-art review of collapsible soils", Science and Technology, Special Review, Sultan Qaboos University, pp.115-135, 2000.

[5] FERREIRA, S.R.M. Colapso e expansão de solos naturais não saturados devidos à inundação, Rio de Janeiro, Tese de D.Sc., COPPE/UFRJ, Rio de Janeiro RJ, 1995.

[6] RAFIE, B.M.A., MOAYED, R.Z., ESMAELI, M., "Evaluation of soil collapsibility potential: A case study of Semnan railway station", Electronic Journal of Geotechnical Engineering, v.13, pp.1-7, 2008.

[7] AYADAT, T. HANNA, A. H. Assessement of soil collapse prediction methods, IJE Transactions B: Aplications. v.25, n.1, pp.19-26, 2012.

[8] CLEMENCE, S.P., FINBARR, A.O.,"Design considerations for collapsible soils", Journal of the Geotechnical Engineering Division, American Society of Civil Engineers, n.107, pp.305-307, 1981.

[9] JENNINGS, J.E. KNIGHT, K. "A guide to construction on or with materials exhibiting additional settlement due to a collapse of grain structure", In: Proceedings of $4^{\text {th }}$ Regional Conference for Africa on Soil Mechanics and Foundation Engineering, Durban, pp.99-105, 1975.

[10] MENDONÇA NETO, H.C., FERREIRA, S.R.M. "Behavior of volume change due to wetting in a collapsible soil to use in irrigation channel", Journal of Civil Engineering and Architecture., v.2, pp.770 778, 2015.

[11] ARAGÃO, C. J. G., MELO, A. C., "Fundações Rasas em solo colapsível: um caso no semi-árido de Pernambuco", In: VII Congresso Brasileiro de Mecânica dos Solos e Engenharia de Fundações, pp.19-40, Olinda, v.2, 1982.

[12] FUCALE, S. P., Comportamento de Variação de Volume Devido à Inundação em Alguns Solos Colapsíveis do Estado de Pernambuco, Dissertação de M.Sc., UFPE, Recife, PE, Brasil, 2000.

[13] SILVA, M. J. R., Comportamento geomecânico de solos colapsíveis e expansivos em Petrolina-PE: Cartas de Suscetibilidade, Dissertação de M.Sc., UFPE, Recife, PE, Brasil, 2003.

[14] FERREIRA, S.R.M. FUCALE, S.P., "Evaluation of the collapsibility of coils in the semiarid region of Pernambuco", Brazil, Journal of Civil Engineering and Architecture, v.8, n.10, pp.1285-1292, 2014.

[15] TORRES, M. S. Avaliação da colapsibilidade e da resistência de ponta de um solo de Petrolina devido à inundação, Dissertação de M.Sc.,UFPE, Recife, PE, Brasil, 2014.

[16] FORTUNATO, E.M.C. Renovação de plataformas ferroviárias: estudos relativos à capacidade de carga. Porto: Tese de Doutorado, Universidade do Porto - Faculdade de Engenharia, 2005.

[17] SANTOS, P.J.C.R. Análise estrutural de pavimento utilizando LWD. Dissertação de M.Sc., Instituto Militar de Engenharia, Rio de Janeiro, RJ, Brasil, 2014.

[18] PREUSSLER, L.A. Contribuição ao estudo da deformabilidade de camadas de pavimento. Dissertação de M.Sc., Escola Politécnica da Universidade de São Paulo, São Paulo, SP, Brasil, 2007. 
[19] JUSTO, J.L.A., DELGADO, A., RUIZ, J. “The influence of stress-path in the collapse - swelling of soils at the laboratory", In: Proc. $5^{\text {th }}$ International Conference on Expansive Soils, Adelaide, v.1, pp. 67-71, 1984.

[20] GENS A., ALONSO E.E., "A framework for the behaviour of unsaturated expansive clays", Canadian Geotechnical Journal, v. 29, pp. 1013-1032, 1992.

[21] ASSOCIAÇÃO BRASILEIRA DE NORMAS TÉCNICAS. NBR 6457: “amostras de solo - preparação para ensaios de compactação e ensaios de caracterização”, Rio de Janeiro, ABNT, 2016. 10p.

$[22]$ . NBR 7181: “solo - análise granulométrica - procedimento.” Rio de Janeiro, ABNT, 2016. 9p.

[23] _. NBR 6548: "solo - grãos de pedregulho retidos na peneira de 4,8 mm: determinação da massa especifica e da absorção de água. Rio de Janeiro, ABNT, 2016, 10p.

[24] ROAD AND TRANSPORT RESEARCH ASSOCIATION, Working Group for Foundation and Soils Engineering. Dynamic Plate-Load Testing with the Aid of the Light Drop-Weight TesterTechnical, Test Code for Soil and Rock Mechanics in Road Constructions TP BF-StB Part B 8.3, German, 2003.

[25] TERRATEST GMBH. Instruction Manual Light Weight Deflectometer for the dynamic plate load test, 2013.

[26] FLEMING, P., FROST, M., LAMBERT, J. Lightweight deflectometers for quality assurance in road construction, Bearing Capacity of Roads; Railways and Airfields, eds Tutumluer and Al-Qadi, pp. 809-818, 2009. 